combination of $a$ 's yields the numerical coefficient for that combination. For example, in the summation for $S_{11}$ given in Table $2,1 / a_{0}{ }^{4}$ has as the $a_{1}{ }^{k-1} a_{n-k+1}$ coefficient $a_{1}{ }^{3} a_{8}$ which, in turn, has the numerical coefficient 11 . The $a_{1}^{2} a_{3} a_{6}$ combination has $2 ! \times 1 ! \times 1$ ! as the product of the factorials of the exponents. If $11 \times 3 ! \times 1$ ! is divided by $2 ! \times 1 ! \times 1$ !, the result is 33 which is the numerical coefficient of the $a_{1}^{2} a_{3} a_{6}$ combination.

Construction of Table 1. Table 1 shows the number of coefficients of $-1 / a_{0}$ as the extreme right-hand entry corresponding to a given $n$. The next entry to the left is the number of coefficients for $+1 / a_{0}^{2}$, etc. The pattern for the number of $-1 / a_{0}$ and $+1 / a_{0}{ }^{2}$ coefficients is obvious. It should also be noted that entries to the left of the stepped division remain fixed for $n$ and $k$. The entries in a column for $k=(n-h)$, where $h=0,1,2$, etc., are formed from cumulative sums, starting from the right, of the entries in the row for $n=h$. For example, for $n=6, h=5$, and $k=1$, the entry is 1 . This entry is equal to the first 1 on the right in the row for $n=5$. For $n=7, h=5$, and $k=2$, the entry is 3 . This entry is equal to $(1+2)$ which is the sum of the first two right-hand entries of the row for $n=5$. A column is formed in this fashion until the sum of the entries in the row (in this case, the fifth) is arrived at, after which the entries in the column remain fixed at this value. The fixed values are those to the left of the stepped division, as explained above.

Summary. The method of finding $S_{n}$ may be summarized briefly as follows. First, obtain the number of coefficients of each $(-1)^{k} / a_{0}{ }^{k}$ from Table 1 or an extension thereof. Second, by some systematic procedure obtain as many different combinations of the $a$ 's as there are coefficients specified by Table 1, making certain that the weight of each combination equals $n$ and the degree equals $k$. Third, find $n \times(k-1)$ ! divided by the product of the factorials of the exponents to obtain the numerical value of a combination. A listing of $S_{n}$ from $n=1$ to $n=11$ is given in Table 2 .

Georgia Institute of Technology

Atlanta, Georgia

1. E. P. AdAMs, Smithsonian Mathematical Formulae and Tables of Elliptic Functions, Smithsonian Institution, Washington, 1947, p. 2-3.

2. G. ChRYstal, Textbook of Algebra, Vol. 1, Chelsea Publishing Co., New York, 1952, p. 434.

3. G. Chrystal, Textbook of Algebra, Vol. 2, Chelsea Publishing Co., New York, 1952, p. 558.

\title{
An Alternating Direction Method for Solving The Biharmonic Equation
}

\author{
By S. D. Conte and R. T. Dames
}

1. Introduction. The biharmonic operator, defined by

$$
\Delta \Delta=\frac{\partial^{4}}{\partial x^{4}}+2 \frac{\partial^{4}}{\partial x^{2} \partial y^{2}}+\frac{\partial^{4}}{\partial y^{4}},
$$

plays a role in the classical theory of plates similar to that of the Laplace operator in potential theory. In particular, the biharmonic equation, $\Delta \Delta W=0$, together

Received 4 October 1957. 
with appropriate boundary conditions, describes the small deflections in a thin homogeneous plate under various forces exerted on the plate boundary. From a computational point of view, direct numerical solutions of such boundary value problems are of considerable interest even in those cases where analytic solutions in series form are available.

The use of relaxation methods for the solution of the biharmonic equation has been considered by Frankel [3] and more recently by Held, Heller and Lubell [2]. It is the purpose of this paper to present an implicit alternating direction iterative scheme which appears to be more efficient than any of the known relaxation methods. The procedure is an extension of a method developed by Douglas and Rachford [1] for Poisson's equation.

2. The differential and difference equations. For definiteness we shall consider the problem of determining the deflections $W$ in a square homogeneous plate supported along its boundaries and bent by moments distributed along two opposite edges. The boundary value problem associated with $W$ may be written :

$$
\begin{aligned}
& \text { (a) } \quad \Delta \Delta W=\frac{\partial^{4} W}{\partial x^{4}}+2 \frac{\partial^{4} W}{\partial x^{2} \partial y^{2}}+\frac{\partial^{4} W}{\partial y^{4}}=0 \quad(x, y) \in R \text {, } \\
& \text { (b) } W(x, y)=\frac{\partial^{2} W}{\partial x^{2}}=0 \text { at } x=0,1 \text { for } 0<y<1 \text {, } \\
& \text { (c) } W(x, y)=0 \quad \text { at } y=0,1 \text { for } 0<x<1 \text {, } \\
& \text { (d) } \frac{\partial^{2} W}{\partial y^{2}}=f(x) \quad \text { at } y=0 \quad \text { for } 0<x<1 \text {, } \\
& \text { (e) } \frac{\partial^{2} W}{\partial y^{2}}=g(x) \quad \text { at } y=1 \quad \text { for } 0<x<1 \text {, }
\end{aligned}
$$

where $R$ is the open region $(0<x<1,0<y<1)$ and $f(x)$ and $g(x)$ describe the bending moments.

We superimpose a square mesh over the region $R$ with grid size $h=\frac{1}{M}$, where $M$ is a positive integer, and employ the following notation:

and

$$
\begin{aligned}
w(i h, j h) & =w_{i, j}, \\
\nabla_{x}^{2} w_{i, j} & =w_{i+1, j}-2 w_{i, j}+w_{i-1, j},
\end{aligned}
$$

$$
\nabla_{x}^{4} w_{i, j}=w_{i+2, j}-4 w_{i+1, j}+6 w_{i, j}-4 w_{i-1, j}+w_{i-2, j} .
$$

The usual finite difference approximations to (2) are :
(a) $\nabla_{x}^{4} w_{i, j}+2 \nabla_{y}^{2} \nabla_{x}^{2} w_{i, j}+\nabla_{y}^{4} w_{i, j}=0 \quad(i h, j h) \in R^{\prime}$
(b) $\quad w_{i, j}=0$
$(j=1,2, \cdots M-1)$,
(c) $w_{i, 0}=w_{i, M}=0$
$(i=1,2, \cdots M-1)$,
(d) $\quad w_{i, 1}=-w_{i,-1}+h^{2} f(i h)$
$(i=1,2, \cdots M-1)$,
(e) $w_{i, M+1}=-w_{i, M-1}+h^{2} g(i h)$
$(i=1,2, \cdots M-1)$,

where $R^{\prime}$ is the set of lattice points $(i h, j h)$ in $R$. 
Since formulation (3) represents a system of $(M-1)^{2}$ equations in $(M-1)^{2}$ unknowns, the amount of computing time involved in solving (3) will be impractical for large $M$ unless one can employ a numerical method which avoids the use of $(M-1)^{2}$ th order systems. Even when the usual point relaxation methods are used, they converge very slowly. Thus for the solution of (3) we consider the following iteration scheme which involves line rather than point relaxation:

(a) $u_{i, j}^{(n+1)}=u_{i, j}^{(n)}-r_{n+1} \nabla_{x}^{4} u_{i, j}^{\left(n+\frac{1}{j}\right)}-2 r_{n+1} \nabla_{y}^{2} \nabla_{x}^{2} u_{i, j}^{(n)}-r_{n+1} \nabla_{y}^{4} u_{i, j}^{(n)}$,

(b) $u_{i, j}^{(n+1)}=u_{i, j}^{(n+1)}-r_{n+1} \nabla_{y}^{4} u_{i, j}^{(n+1)}+r_{n+1} \nabla_{y}^{4} u_{i, j}^{(n)} \quad(i h, j h) \in R^{\prime}$,

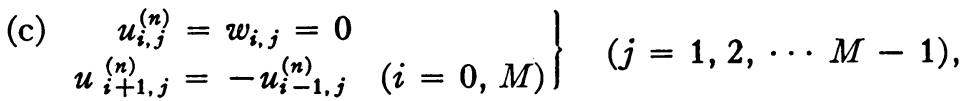

(d) $\quad u_{i, j}^{(n)}=w_{i, j}=0 \quad(j=0, M) \quad(i=1,2, \cdots M-1)$,

(e) $\left.\begin{array}{rl}u_{i, 1}^{(n)} & =-u_{i,-1}^{(n)}+h^{2} f(i h) \\ u_{i, M+1}^{(n)} & =-u_{i, M-1}+h^{2} g(i h)\end{array}\right\} \quad(i=1,2, \cdots M-1)$.

Here $r_{n+1}$ is an iteration parameter which will be chosen to accelerate convergence and $u_{i, j}^{(o)}$, for $(i h, j h)$ in $R^{\prime}$, is an initial approximation to $w_{i, j}$.

Equation $4(\mathrm{a})$ is implicit in $x$ alone and represents a system of $M-1$ equations in $M-1$ unknowns along a single line in the $x$-direction. A similar statement can be made for equation $4(\mathrm{~b})$ and the variable $y$. Hence, the numerical procedure for executing one iteration consists of first solving 4 (a) for $u_{i, j}^{(n+1)}$ at all points in $R^{\prime}$ and subsequently solving $4(\mathrm{~b})$ for $u_{i, j}^{(n+1)}$. At every stage of this iteration scheme, the matrices involved are all of order $M-1$ with at most 5 non-zero elements in any row (either on the main diagonal or on two adjacent diagonals). Such "quidiagonal" systems can be solved efficiently by an extension of an algorithm for solving tridiagonal matrices due to L. H. Thomas and will be discussed later in Section 5.

3. Convergence of the iterative procedure. To study the convergence of $u_{i, j}^{(n)}$ to $w_{i, j}$, it is convenient to eliminate $u_{i, j}^{\left(n+\frac{1}{j}\right)}$ from 4 (a) and $4(\mathrm{~b})$. This yields the single equation

$u_{i, j}^{(n+1)}=u_{i, j}^{(n)}-r_{n+1}\left(\nabla_{x}^{4} u_{i, j}^{(n+1)}+\nabla_{y}^{4} u_{i, j}^{(n+1)}+2 \nabla_{y}^{2} \nabla_{x}^{2} u_{i, j}^{(n)}\right)-r_{n+1}^{2} \nabla_{x}^{4} \nabla_{y}^{4}\left(u_{i, j}^{(n+1)}-u_{i, j}^{(n)}\right)$.

Now let the error $e_{i, j}^{(n)}$ be defined by

$$
e_{i, j}^{(n)}=u_{i, j}^{(n)}-w_{i, j}
$$

Then $e_{i, j}^{(n)}$ satisfies the following equation.

$$
\begin{aligned}
e_{i, j}^{(n+1)}=e_{i, j}^{(n)}-r_{n+1}\left(\nabla_{x}^{4} e_{i, j}^{(n+1)}+\nabla_{y}^{4} e_{i, j}^{(n+1)}+\right. & \left.2 \nabla_{y}^{2} \nabla_{x}^{2} e_{i, j}^{(n)}\right) \\
& -r_{n+1}^{2} \nabla_{x}^{4} \nabla_{y}^{4}\left(e_{i, j}^{(n+1)}-e_{i, j}^{(n)}\right), \quad(i h, j h) \in R^{\prime}
\end{aligned}
$$

together with homogeneous boundary conditions corresponding to relations 4 (c), $4(\mathrm{~d})$ and $4(\mathrm{e})$.

The eigenfunctions of (5) are of the form

Now suppose

$$
e_{i, j}^{(n)}=A \sin (p \pi i h) \sin (q \pi j h) .
$$

$$
e_{i, j}^{(n+1)}=\lambda_{p, q} e_{i, j}^{(n)}, \quad p, q=1,2, \cdots M-1
$$


By substituting this expression into (5) we find that

$$
\lambda_{p, q}\left(\alpha_{n+1}\right)=\frac{\left(1-\alpha_{n+1} S_{p}^{2} S_{q}^{2}\right)^{2}}{1+\alpha_{n+1}\left(S_{p}^{4}+S_{q}^{4}\right)+\alpha^{2}{ }_{n+1} S_{p}^{4} S_{q}^{4}},
$$

where we have set

$$
\begin{aligned}
\alpha_{n+1} & =16 r_{n+1} \\
S_{p} & =\sin \frac{p \pi h}{2}
\end{aligned}
$$

and

$$
S_{q}=\sin \frac{q \pi h}{2}
$$

Since the initial error $e_{i, j}^{(o)}$ can be expanded in the form

$$
e_{i, j}^{(o)}=\sum_{p, q=1}^{M-1} C_{p, q}^{(o)} \sin (p \pi i h) \sin (q \pi j h)
$$

we have

$$
e_{i, j}^{(n)}=\sum_{p, q-1}^{M-1} C_{p, Q}^{(n)} \sin (p \pi i h) \sin (q \pi j h)
$$

where

$$
C_{p, q}^{(n)}=\prod_{k=1}^{n} \lambda_{p, q}\left(\alpha_{k}\right) C_{p, q}^{(o)}
$$

For all positive $\alpha_{k}$, it is clear from (6) that $0 \leq \lambda_{p, q}\left(\alpha_{k}\right)<1$ for all $p$ and $q$. Thus, after $n$ iterations each component of the error decreases by a factor of

$$
\prod_{k=1}^{n} \lambda_{p, q}\left(\alpha_{k}\right)
$$

as long as $\alpha_{k}$ (and hence $r_{k}$ ) is positive. The minimum value of $\lambda_{p, q}(\alpha)$ is zero and occurs when

$$
\alpha=\frac{1}{S_{p}^{2} S_{q}^{2}}
$$

This implies, at least theoretically, that after $\frac{M(M-1)}{2}$ iterations, with appropriate choices for the $\alpha_{k}$, one could make all components of the error vanish and thus obtain the exact solution. In practice, however, it is more efficient to reduce all components of the error by a fraction of their original value. With this in mind, we seek a method for choosing the $\alpha_{k}$ which optimizes the convergence rate of the iterative procedure.

Let

$$
\tilde{\lambda}_{p, q}(\alpha) \equiv\left(\frac{1-\alpha S_{p}{ }^{2} S_{q}{ }^{2}}{1+\alpha S_{p}{ }^{2} S_{q}{ }^{2}}\right)^{2}
$$


Since $S_{p}{ }^{4}+S_{q}{ }^{4} \geq 2 S_{p}{ }^{2} S_{q}{ }^{2}$ it follows from (6) that

$$
\lambda_{p, q}(\alpha) \leq \lambda_{p, q}(\alpha) \text {. }
$$

In view of this inequality, any convergence results based on $\lambda_{p, q}(\alpha)$ will also apply when $\lambda_{p, q}(\alpha)$ is replaced by $\lambda_{p, q}(\alpha)$. In what follows we shall base our considerations on the more convenient quantity $\lambda_{p, q}$.

4. Determination of the iteration parameters. We next consider the problem of finding a set of iteration parameters $\alpha_{k}(k=1,2, \cdots t)$ such that the maximum of the function

$$
G_{t}\left(S_{p}{ }^{2} S_{q}^{2}, \alpha_{k}\right)=\prod_{k=1}^{t} \tilde{\lambda}_{p, q}\left(\alpha_{k}\right)=\prod_{k=1}^{t}\left(\frac{\alpha_{k}{ }^{-1}-S_{p}{ }^{2} S_{q}{ }^{2}}{\alpha_{k}{ }^{-1}+S_{p}{ }^{2} S_{q}{ }^{2}}\right)^{2}
$$

is a minimum for $p$ and $q$ in the range $1,2, \cdots M-1$. This problem may be resolved efficiently by treating it as a Ghebyshev minimax problem and is discussed in [4]. Applying the results obtained there to the function $G_{t}\left(S_{p}{ }^{2} S_{q}{ }^{2}, \alpha_{k}\right)$ we find that a recommended set of iteration parameters is

$$
\alpha_{k}=x^{1-k}, \quad k=1,2, \cdots t
$$

for $0<x<1$. This gives an upper bound on $G_{t}\left(S_{p}{ }^{2} S_{q}{ }^{2}, \alpha_{k}\right)$ defined by

$$
G_{t}\left(S_{p}^{2} S_{q}^{2}, \alpha_{k}\right) \leq P_{t}(x) \equiv\left(\frac{1-x^{4}}{1+x^{4}} e^{-\frac{x^{4}}{1-x}}\right)^{4}
$$

provided that

$$
x^{t-1} \leq S_{1}^{4} \text { and } \frac{S_{1}{ }^{4}}{S^{4} M-1} \ll 1 .
$$

The iteration parameters (7) are not asserted to be the best possible set. A measure of the loss of convergence due to not using the set of optimum parameters $\bar{\alpha}_{k}(k=1,2, \cdots, t)$ is given by

$$
\frac{G_{t}\left(S_{p}^{2} S_{q}^{2}, \bar{\alpha}_{k}\right)}{G_{t}\left(S_{p}^{2} S_{q}^{2}, \alpha_{k}\right)} \approx e^{-\frac{2 x^{4}}{1-x}}
$$

The formal process of choosing the iteration parameters $\alpha_{k}$ proceeds as follows : First one decides on a value of $P_{t}(x)$. When the initial error is to be reduced by a given factor, the choice of $P_{t}(x)$ determines the number of cycles necessary for this reduction. Next, $x$ is determined from (8) while the number $t$ of iterations per cycle is computed by solving (9) for $t$; i.e.,

$$
t \geq 1+\frac{4 \log S_{1}}{\log x}
$$

Hence, after one cycle, consisting of $t$ double sweeps, every component of the initial error will be reduced by a factor of $P_{t}(x)$.

The significance of relation (10) is important for the initial selection of $P_{t}(x)$ and hence $x$. In order to carry out the iteration procedure in the most efficient manner, the value of $x$ should be small enough so that this ratio is close to unity. Although a decrease in $x$ means an increase in $P_{t}(x)$, it still may be more efficient in terms of total sweeps, to increase the number of cycles while at the same time 
decreasing the number of iterations within a cycle. At any rate, for this scheme to give the best results, $P_{t}(x)$ should be such that $x<0.2$. For this range

$$
\frac{G_{t}\left(S_{p}{ }^{2} S_{q}{ }^{2}, \bar{\alpha}_{k}\right)}{G_{t}\left(S_{p}{ }^{2} S_{q}{ }^{2}, \alpha_{k}\right)}>0.8 .
$$

As an example of the method, consider the solution of system (4) using a $20 \times 20$ grid. Suppose we desire to reduce the initial error by a factor of $10^{-6}$. We choose $x=0.2$ so that $P_{t}(x) \simeq 0.01$. This means that 3 cycles are necessary for the reduction. The number of double sweeps per cycle is given by

$$
t \sim 1+\frac{4 \log \sin \frac{\pi}{40}}{\log .2} \simeq 7.35 \text {. }
$$

Hence, it takes three cycles of eight iterations each, using

$$
r_{k}=\frac{(0.2)^{1-k}}{16}, \quad k=1,2, \cdots 8
$$

to reduce the error by approximately $10^{-6}$. This involves 48 single sweeps over the grid.

5. The solution of the quidiagonal systems. The quidiagonal systems arising from the iteration procedure (4) may be written in the general form :

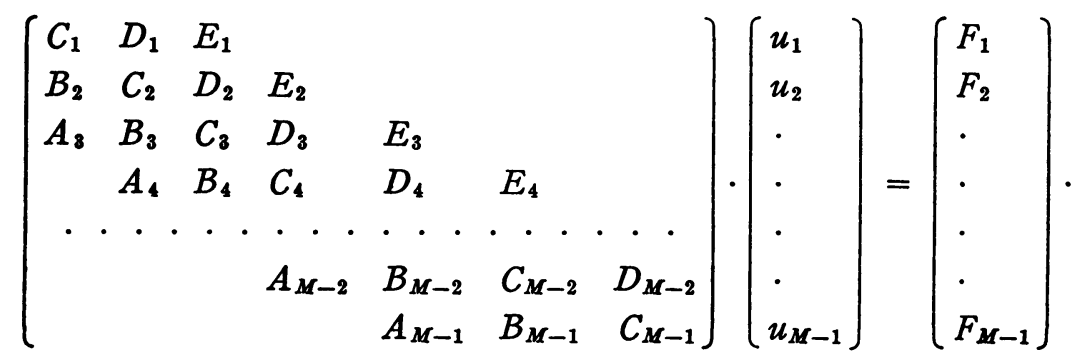

Such systems may be solved by the following elimination scheme: Let

$$
\begin{array}{llrl}
\omega_{1}=C_{1}, & & \\
\beta_{1}=D_{1} / \omega_{1}, & \beta_{0}=0, & \beta_{M-1}=0, \\
\gamma_{1}=E_{1} / \omega_{1}, & \gamma_{0}=0, & \gamma_{M-1}=\gamma_{M-2}=0,
\end{array}
$$

and define recursively

$$
\begin{aligned}
& \delta_{j}=B_{j}-A_{j} \beta_{j-2}, \\
& \omega_{j}=C_{j}-A, \gamma_{j-2}-\delta_{j} \beta_{j-1}, \quad \text { for } 2 \leq j \leq M-1, \\
& \beta_{j}=\left(D_{j}-\delta_{j} \gamma_{j-1}\right) / \omega_{j} \\
& \gamma_{j}=E_{j} / \omega_{j} .
\end{aligned}
$$

Next form

$$
\begin{aligned}
& h_{0}=0 \\
& h_{1}=F_{1} / \omega_{1}, \\
& h_{j}=\left(F_{j}-A_{j} h_{j-2}-\delta_{j} h_{j-1}\right) / \omega_{j}, \quad 2 \leq j \leq M-1 .
\end{aligned}
$$


The values of $u$ are then obtained successively from the formulas

$$
\begin{gathered}
u_{M-1}=h_{M-1}, \\
u_{j}=h_{j}-\beta_{j} u_{j+1}-\gamma_{j} u_{j+2}, \quad j=M-2, M-3, \cdots 1 .
\end{gathered}
$$

For the matrices arising from equations 4 (a) and 4 (b) we have

$$
\begin{array}{ll}
B_{j}=D_{j}=-4 & \text { for all } j, \\
A_{j}=E_{j}=1 & \text { for all } j,
\end{array}
$$

while the diagonal elements depend on the iteration parameters $r_{k}$. The components $F_{j}$ on the right hand side of the general system (12) will, in our case, involve values of $u$ obtained on the previous iteration and may be evaluated in a straightforward manner. When relaxing along lines in the $y$-direction or along either of the lines $y=h, y=(M-1) h$, these components will also require values of $f(x)$ and $g(x)$. For one complete iteration (i.e., a double sweep over the grid) one must solve $2(M-1)$ systems of the form (12) with each system of order $M-1$. Using the elimination scheme described above, this takes approximately $30(M-1)^{2}$ additions, $13(M-1)^{2}$ multiplications and $6(M-1)^{2}$ divisions. In arriving at this estimate, we have made use of relations (13). Regarding both a multiplication and a division as equivalent timewise to 5 additions, we may estimate the total time $T$ for one iteration as $25(M-1)^{2}$ where $\tau$ is the average time in seconds for one multiplication. For a computer capable of executing $\mathbf{4 0 0 0}$ multiplications per second we have approximately

$$
T=6.25 \times 10^{-3}(M-1)^{2} \text { seconds. }
$$

Thus, in the example at the end of Section 4 , it would take approximately 54 seconds to reduce the initial error by a factor of $10^{-6}$.

6. Remarks. The particular fourth order problem considered here can, of course, be solved by more direct methods. The second-order Richardson method seems to have been successfully used although it has recently been shown by E. Windsor [5] that the unextrapolated Richardson Method does not converge. Another successful approach has been to reformulate the fourth order equation as two coupled second order elliptic equations which can then be solved by standard methods.

On the basis of the theory presented here, the alternating direction method seems to be decidedly superior to other proposed relaxation methods, the factor of superiority generally increasing as the number of mesh points increases.

The method proposed here for solving the biharmonic equation can readily be adapted to the case of rectangular regions with other boundary conditions. However, for arbitrary regions and for general boundary conditions, the proof given here does not hold and hence convergence is not assured.

Digital Computing Center

The Ramo-Wooldridge Corporation

P. O. Box 45564, Airport Station

Los Angeles 45, California

1. J. Douglas, JR. \& H. H. Rachford, JR., "On the numerical solution of heat conduction problems in two and three space variables," Amer. Math. Soc., Trans., v. 82, 1956, p. 421-439.

2. M. Held, J. Heller, \& D. LubEll, "Rates of convergence of successive iteration schemes for fourth order elliptic difference equations," New York University. (To be published). 
3. S. P. FRANKEL, "Convergence rates of iterative treatments of partial differential equations," $M T A C$, v. IV, 1950 , p. 65-75.

4. E. L. WACHSPRESS, "Cure: a generalized two space-dimension multigroup coding for the IBM 704," KAPL-1724, AEC Research and Development Report, Physics and Math. (TID-4500, 13 Ed.), Knolls Atomic Power Lab., Schenectady, N. Y.

5. E. WINDSOR, "Iterative solutions of biharmonic differential equations," New York University, Master's Thesis, May, 1957.

\section{TECHNICAL NOTES AND SHORT PAPERS}

\section{On The Numerical Evaluation of Cauchy Principal Values of Integrals}

\section{By I. M. Longman}

Introduction. This note demonstrates a simple method for the numerical evaluation of the Cauchy principal value of an integral

$$
P \int_{a}^{b} f(x) d x \quad(x \text { real })
$$

when the integrand $f(x)$ has singularities at one or more points in the finite interval $[a, b]$ of integration. The necessity for such numerical evaluation arises sometimes in the solution of problems in applied mathematics. Such integrals can occur, for example, in the solution of wave propagation problems. For instances of this see Lamb [1], and Pekeris [2]. Sometimes a singularity can be avoided by a suitable transformation in the complex plane. The present note presents a simple direct method of dealing with such a singularity.

Formulation of the method. The method is quite general and can be applied separately to each singularity of $f(x)$, and so, for simplicity, we will suppose that $f(x)$ has only one singularity which, without loss of generality, we may suppose to be at $x=0$. Suppose then we require to evaluate by some numerical quadrature method the integral

$$
I=P \int_{-a}^{a} f(x) d x
$$

where the range of integration is made symmetrical about the origin by splitting up the integral into two ranges if necessary. We split up the function $f(x)$ into its odd and even components

$$
f(x)=\frac{1}{2}[f(x)-f(-x)]+\frac{1}{2}[f(x)+f(-x)]=g(x)+h(x), \text { say. }
$$

Then

$$
I=P \int_{-a}^{a} g(x) d x+P \int_{-a}^{a} h(x) d x .
$$

Now since $g(x)$ is an odd function the first integral vanishes, and so

$$
I=2 \int_{0}^{a} h(x) d x
$$

where either there is now no singularity at $x=0$, or, if there is, it is not such as to make the integral (4) diverge, since we suppose the Cauchy principal value

Received 18 March 1958. This work was done while the author was working at The Weizmann Institute of Science, Rehovot, Israel. 